\title{
Metrics with distributional curvature
}

\author{
David Garfinkle * \\ Department of Physics, Oakland University, Rochester, Michigan 48309
}

\begin{abstract}
This paper considers metrics whose curvature tensor makes sense as a distribution. A class of such metrics, the regular metrics, was defined and studied by Geroch and Traschen. Here, we generalize their definition to form a wider class: semi-regular metrics. We then examine in detail two metrics that are semi-regular but not regular: (i) Minkowski spacetime minus a wedge and (ii) a certain traveling wave metric.
\end{abstract}

04.20.-q,04.20.Cv

\section{INTRODUCTION}

In electrodynamics, one often treats charges and currents that are confined to points, curves or surfaces. These idealized sources are supposed to represent objects where the charge or current is very concentrated. The mathematical description for such concentrated sources, as well as the electric and magnetic fields that they give rise to, is the theory of distributions. In electrodynamics, the use of distributions is straightforward because Maxwell's equations are linear. The sum of distributions or the derivative of a distribution is well defined. However, in general the product of two distributions is not well defined. This makes the use of distributions in a nonlinear theory problematic.

In general relativity, one would like to have a treatment of concentrated sources. Since the Einstein field equations are nonlinear, one cannot simply assume that the metric is a distribution, since then the Einstein tensor would, in general, not be well defined, and the field equations would not make sense. The solution is to identify a class of metrics whose curvature is well defined as a distribution. (Alternatively, one could consider generalized functions [1]:2] instead of distributions. This approach has been used; 3 5] but we will not consider it here).

For sources on thin shells, an appropriate class of metrics has been identified. [6 8] Here the class consists of metrics that are smooth on two manifolds with timelike boundary and where the induced metrics on the two boundaries agree. Identification of the boundaries then produces a metric whose curvature makes sense as a distribution. A similar formalism can be used to make sense of thin null shells. [9 12]

One would like to have a large class of metrics whose curvature makes sense as a distribution. This class would include the thin shell metrics, but would not include all distributional metrics. Such a class was defined by Geroch and Traschen [13] and called regular metrics. (These metrics will be discussed in more detail in the next section). One of the results of reference [13] is that if the curvature of a regular metric is concentrated on a surface in spacetime, then the surface must have dimension 3. In other words, regular metrics can represent thin shells but not thin strings.

Though the class of regular metrics is quite wide, there remains the possiblility that one can find a wider class. That is, there may be metrics that are not regular in the sense of reference [13] but whose curvature makes sense as a distribution. An indication that this is the case is provided by an examination of the traveling wave metrics. 14] These metrics have the form $\tilde{g}_{a b}=g_{a b}+F k_{a} k_{b}$ where $g_{a b}$ is a metric with null, hypersurface orthogonal Killing vector $k^{a}$ and $F$ is a scalar. The expression for the curvature of $\tilde{g}_{a b}$ is linear in $F$. Thus, provided that $F$ is any scalar that is a distribution, it seems that the curvature of $\tilde{g}_{a b}$ should be well defined as a distribution. This is true even for an $F$ for which $\tilde{g}_{a b}$ is not regular. (One can also make this sort of argument using the generalized Kerr-Schild metrics, [15] which have the form $\tilde{g}_{a b}=g_{a b}+F k_{a} k_{b}$ where $k^{a}$ is a null, shear-free, geodetic congruence on $g_{a b}$ ).

This paper defines and studies a class of metrics, which we call semi-regular metrics, which is wider than the class of regular metrics and whose curvature makes sense as a distribution. Section 2 gives the definition of semi-regular metrics. The next two sections examine in detail two metrics that are semi-regular but not regular: with Minkowski spacetime minus a wedge in section 3 and a certain traveling wave metric in section 4 .

*Email: garfinkl@oakland.edu 


\section{SEMI-REGULAR METRICS}

To define regular metrics, Geroch and Traschen begin with an expression for the Riemann tensor of a smooth metric. Let $\nabla_{a}$ be any smooth derivative operator with Riemann tensor $R_{a b c}{ }^{d}$ and let $\tilde{g}_{a b}$ be any smooth metric. Define the Christoffel tensor

$$
C_{a b}^{c} \equiv \frac{1}{2}\left(\tilde{g}^{-1}\right)^{c d}\left(\nabla_{a} \tilde{g}_{b d}+\nabla_{b} \tilde{g}_{a d}-\nabla_{d} \tilde{g}_{a b}\right)
$$

Then the Riemann tensor of $\tilde{g}_{a b}$ is

$$
\tilde{R}_{a b c}{ }^{d}=R_{a b c}{ }^{d}+2 \nabla_{[b} C_{a] c}^{d}+2 C_{m[b}^{d} C_{a] c}^{m} .
$$

The idea is to use equation (2) as the definition of curvature. The class of regular metrics is then defined in such a way that the quantities in equation (2) make sense as distributions.

In this paper we use the definition of tensor distribution given by Geroch and Traschen. (For a different approach see reference [16]). We now recall some of the basic definitions. (For more detail, see reference 13]). A test field is a smooth tensor density of compact support. A tensor distribution is a continuous linear map from the vector space of test fields of a given index structure to the real numbers. A tensor field $\mu_{a \ldots c} b \ldots d$ defined almost everywhere is called locally integrable if for every test field $t^{a \ldots c} b_{b \ldots d}$ the scalar density $\mu_{a \ldots c} b \ldots d t^{a \ldots c} b_{b .}$ is Lebesgue measurable and its Lebesgue integral converges. Note that through this integral, every locally integrable tensor field defines a distribution. A tensor field is called locally square integrable provided that its outer product with itself is locally integrable. A tensor field $\mu_{a \ldots c}{ }^{b \ldots d}$ is called locally bounded provided that the scalar density $\mu_{a \ldots c}{ }^{b \ldots d} t^{a \ldots c} b \ldots d$ is bounded for all test fields $t^{a \ldots c} b_{b .}$. For any smooth derivative operator $\nabla_{a}$ the derivative of a distribution $S_{a \ldots c} \ldots d$ is the distribution $\nabla_{e} S_{a \ldots c}{ }^{b \ldots d}$ such that

$$
\nabla_{e} S_{a \ldots c} b \ldots d\left[t^{e a \ldots c} b \ldots d\right]=-S_{a \ldots c} b \ldots d\left[\nabla_{e} t^{e a \ldots c} b \ldots d\right]
$$

where $t^{e a \ldots c} \ldots d$ is any test field and square brackets denote the action of the distribution on the test field. Let $\mu_{a \ldots c} b_{b .}$ be a locally integrable tensor field. Then a locally integrable tensor field $W_{e a \ldots c} b \ldots d$ is called the weak derivative of $\mu_{a \ldots c}{ }^{b \ldots d}$ provided that

$$
\int_{M} \mu_{a \ldots c}^{b \ldots d} \nabla_{e} t_{b \ldots d}^{e a \ldots c}=-\int_{M} W_{e a \ldots c}{ }_{b \ldots d}^{b a \ldots c} t_{b \ldots d}
$$

for all test fields $t_{b \ldots c}^{e a \ldots c}$. (Here the subscript $M$ denotes that the integral is over the whole manifold). In other words, $W_{e a \ldots c}{ }^{b \ldots d}$ is the locally integrable tensor field whose distribution is the derivative of the distribution $\mu_{a \ldots c} b \ldots d$. A locally integrable tensor field may or may not have a weak derivative.

The definition of regular metric given in reference [13] is the following: a metric $\tilde{g}_{a b}$ is called regular provided that (i) $\tilde{g}_{a b}$ and $\left(\tilde{g}^{-1}\right)^{a b}$ exist everywhere and are locally bounded, and (ii) the weak derivative of $\tilde{g}_{a b}$ exists and is locally square integrable. The idea behind this definition is that we would like $C_{a b}^{c}$ to be a tensor field that is both locally integrable and locally square integrable. A locally integrable $C_{a b}^{c}$ defines a distribution, and since the derivative of a distribution is a distribution, the second term on the right hand side of equation (2) is a distribution. A locally square integrable $C_{a b}^{c}$ insures that the third term on the right hand side of equation (2) is locally integrable and therefore defines a distribution.

These considerations suggest that one could define a larger class of metrics with distributional curvature by more directly requiring what is needed to make the terms on the right hand side of equation (2) distributions. We therefore define a metric $\tilde{g}_{a b}$ to be a semi-regular metric provided that (i) $\tilde{g}_{a b}$ and $\left(\tilde{g}^{-1}\right)^{a b}$ exist almost everywhere and are locally integrable, and (ii) the weak first derivative of $\tilde{g}_{a b}$ exists and the tensors $C_{a b}^{c}$ and $C_{m[b}^{d} C_{a] c}^{m}$ are locally integrable.

From the definitions, one can show that all regular metrics are semi-regular, but that some semi-regular metrics are not regular. In particular, consider the case of a semi-regular metric for which $C_{a b}^{c}$ is not locally square integrable. Then the tensor field $C_{m b}^{d} C_{a c}^{n}$ cannot be regarded as a distribution. Nonetheless, since $C_{m[b}^{d} C_{a] c}^{m}$ is locally integrable, there is no obstruction to regarding the right hand side of equation (2) as a distribution.

Since a locally integrable tensor field defines a distribution, it follows from the definition that both $\tilde{R}_{a b c}{ }^{d}$ and, by contraction, $\tilde{R}_{a b}$ are distributions for a semi-regular metric.

The class of semi-regular metrics does not include the signature changing metrics studied by several authors 17 32] If the signature changing metric is discontinuous, then its weak derivative does not exist (i.e. the derivative of the distribution $\tilde{g}_{a b}$ is not a tensor field). If $\tilde{g}_{a b}$ is smooth but degenerate on a hypersurface, then $\left(\tilde{g}^{-1}\right)^{a b}$ is not locally integrable. 
For a regular metric, arbitrary products of the Riemann tensor, the metric and the inverse metric are distributions. In particular, this means that the Einstein tensor of a regular metric is a distribution. Under what circumstances is this also true of a semi-regular metric? For a smooth metric we have

$$
\begin{gathered}
\tilde{G}_{a b}=\tilde{R}_{a b}-\frac{1}{2}\left(\tilde{g}^{-1}\right)^{c d} R_{c d} \tilde{g}_{a b}+\left(\tilde{g}^{-1}\right)^{c d} C_{m[c}^{e} C_{e] d}^{m} \tilde{g}_{a b} \\
+\nabla_{[c}\left(C_{e] d}^{e}\left(\tilde{g}^{-1}\right)^{c d} \tilde{g}_{a b}\right)+C_{d[c}^{e} \nabla_{e]}\left(\left(\tilde{g}^{-1}\right)^{c d} \tilde{g}_{a b}\right) .
\end{gathered}
$$

We use equation (5) as the definition of the Einstein tensor for a semi-regular metric. More precisely, given a semi-regular metric $\tilde{g}_{a b}$ we ask whether each term on the right hand side of equation (5) defines a distribution. If the answer is yes, then we say that the Einstein tensor of $\tilde{g}_{a b}$ is given by the right hand side of equation (5). If the answer is no, then we say that $\tilde{g}_{a b}$ has a Ricci tensor but not an Einstein tensor.

In the rest of the paper we will examine two metrics that are semi-regular, but not regular: Minkowski spacetime minus a wedge and a certain traveling wave metric.

\section{MINKOWSKI SPACETIME MINUS A WEDGE}

In this section, we treat the metric whose line element is

$$
d \tilde{s}^{2}=-d t^{2}+d z^{2}+d r^{2}+r^{2} \cos ^{2} \gamma d \phi^{2} .
$$

This is the metric of Minkowski spacetime where a wedge of angular size $\Delta \phi=2 \pi(1-\cos \gamma)$ has been cut out and the spacetime has been identified along the cut. (For a treatment of this metric in the context of $2+1$ dimensional gravity see [33,34 ). The metric outside of a long straight cosmic string is Minkowski spacetime minus a wedge. Therefore, it is tempting to regard equation (6) as the metric of a zero thickness cosmic string. The distributional stress-energy of $\tilde{g}_{a b}$ (if it is well defined) would then tell us about the energy content of the string. In reference [13 it was shown that $\tilde{g}_{a b}$ is not regular. Here, we will show that $\tilde{g}_{a b}$ is semi-regular, and will calculate its Ricci tensor.

We introduce the ordinary Minkowski metric $g_{a b}$ given by

$$
d s^{2}=-d t^{2}+d z^{2}+d r^{2}+r^{2} d \phi^{2}
$$

and choose as our smooth derivative operator $\nabla_{a}$ the one compatible with $g_{a b}$. We lower and raise all tensor indicies with $g_{a b}$ and its inverse. Introduce the axial Killing field $\psi^{a}=(\partial / \partial \phi)^{a}$. Then we have

$$
\begin{gathered}
\tilde{g}_{a b}=g_{a b}-\sin ^{2} \gamma r^{-2} \psi_{a} \psi_{b}, \\
\left(\tilde{g}^{-1}\right)^{a b}=g^{a b}+\tan ^{2} \gamma r^{-2} \psi^{a} \psi^{b} .
\end{gathered}
$$

Thus $\tilde{g}_{a b}$ and its inverse are defined almost everywhere.

To find a distribution, one must find its action on test fields: that is smooth tensor densities of compact support. We will write all such densities as ordinary tensor fields multiplied by the volume element of $g_{a b}$, and will speak loosely of the "test tensor field" $S^{a b}$ when we mean the tensor density $S^{a b} \epsilon_{c d e f}$. Here, it is important to keep in mind that the underlying manifold is $R^{4}$ with Cartesian coordinates $(t, x, y, z)$ where $x=r \cos \phi$ and $y=r \sin \phi$. Smoothness of a tensor field then means smoothness of the Cartesian components as functions of the Cartesian coordinates. In particular, $\psi_{a}=x \partial_{a} y-y \partial_{a} x$ so the components of $r^{-1} \psi_{a}$ are defined for $r \neq 0$ and are bounded.

For a test field $S^{a b}$, we have

$$
\begin{array}{r}
\tilde{g}_{a b} S^{a b}=S-\sin ^{2} \gamma r^{-2} S^{a b} \psi_{a} \psi_{b} \\
=S-\sin ^{2} \gamma\left(\cos ^{2} \phi S^{y y}+\sin ^{2} \phi S^{x x}-\sin \phi \cos \phi\left[S^{x y}+S^{y x}\right]\right) .
\end{array}
$$

Therefore $\tilde{g}_{a b}$ is locally integrable and thus $\tilde{g}_{a b}$ defines a distribution. We denote the action of the distribution $\tilde{g}_{a b}$ on the test tensor field $S^{a b}$ by $\tilde{g}_{a b}\left[S^{a b}\right]$. A similar calculation shows that $\left(\tilde{g}^{-1}\right)^{a b}$ is locally integrable.

We now calculate the weak derivative of $\tilde{g}_{a b}$. That is, we seek a tensor field $W_{c a b}$ defined almost everywhere, such that

$$
\tilde{g}_{a b}\left[\nabla_{c} S^{c a b}\right]=-\int_{M} W_{c a b} S^{c a b}
$$


for all test tensor fields $S^{c a b}$. (Here the subscript $M$ denotes that the integral is over the whole manifold). It turns out that the weak derivative of $\tilde{g}_{a b}$ is simply $\nabla_{c} \tilde{g}_{a b}$ defined at all points where $r \neq 0$. To see this, note that we have

$$
\begin{array}{r}
\tilde{g}_{a b}\left[\nabla_{c} S^{c a b}\right]= \\
\int_{M} \tilde{g}_{a b} \nabla_{c} S^{c a b}=\lim _{\epsilon \rightarrow 0} \int_{r>\epsilon} \tilde{g}_{a b} \nabla_{c} S^{c a b} \\
=\lim _{\epsilon \rightarrow 0} \int_{r>\epsilon} \nabla_{c}\left(\tilde{g}_{a b} S^{c a b}\right)-S^{c a b} \nabla_{c} \tilde{g}_{a b} \\
=\lim _{\epsilon \rightarrow 0}\left[\int_{r=\epsilon} n_{c} \tilde{g}_{a b} S^{c a b}-\int_{r>\epsilon} S^{c a b} \nabla_{c} \tilde{g}_{a b}\right] .
\end{array}
$$

Here, $n_{c}$ is the unit outward pointing normal to the $r=\epsilon$ surface.

Taking the limit as $\epsilon \rightarrow 0$, we find that the first term goes to zero and the second term becomes an integral over the whole manifold. Therefore, we have $W_{c a b}=\nabla_{c} \tilde{g}_{a b}$. However, we have

$$
\nabla_{a} \psi_{b}=r^{-1}\left(\psi_{b} \nabla_{a} r-\psi_{a} \nabla_{b} r\right)
$$

It then follows that

$$
\nabla_{c} \tilde{g}_{a b}=\sin ^{2} \gamma r^{-3} \psi_{c}\left(\psi_{a} \nabla_{b} r+\psi_{b} \nabla_{a} r\right)
$$

This quantity is locally integrable, but not locally square integrable. (Essentially, the reason for this is that $r^{-1} \psi_{a}$ is bounded and $r^{-1}$ is locally integrable, but $r^{-2}$ is not). It is this lack of local square integrability of $\nabla_{c} \tilde{g}_{a b}$ that prevents $\tilde{g}_{a b}$ from being a regular metric.

From equations (11) and (14) it follows that the Christoffel tensor is

$$
C_{a b}^{c}=\sin ^{2} \gamma r^{-3} \nabla^{c} r \psi_{a} \psi_{b}
$$

The Christoffel tensor is locally integrable. Furthermore, since $\psi^{a} \nabla_{a} r=0$, it follows that $C_{m[b}^{d} C_{a] c}^{m}=0$ and therefore it follows trivially that $C_{m[b}^{d} C_{a] c}^{m}$ is locally integrable. It then follows that $\tilde{g}_{a b}$ is a semi-regular metric.

We now calculate the Ricci tensor of $\tilde{g}_{a b}$. Contracting equation (2) we find that for any semi-regular metric

$$
\tilde{R}_{a b}=R_{a b}+2 \nabla_{[c} C_{a] b}^{c}+2 C_{m[c}^{c} C_{a] b}^{m}
$$

Specializing to the metric of equation (6) and using $C_{c b}^{c}=0$ we then find

$$
\tilde{R}_{a b}=\nabla_{c} C_{a b}^{c} .
$$

Recall that this is a distributional equation which should be interpreted as follows: For any test field $S^{a b}$ we have

$$
\tilde{R}_{a b}\left[S^{a b}\right]=-\int_{M} C_{a b}^{c} \nabla_{c} S^{a b} .
$$

We then find

$$
\begin{array}{r}
\tilde{R}_{a b}\left[S^{a b}\right]=-\lim _{\epsilon \rightarrow 0} \int_{r>\epsilon} C_{a b}^{c} \nabla_{c} S^{a b} \\
=-\lim _{\epsilon \rightarrow 0} \int_{r>\epsilon} \nabla_{c}\left(C_{a b}^{c} S^{a b}\right)-S^{a b} \nabla_{c} C_{a b}^{c} \\
=-\lim _{\epsilon \rightarrow 0}\left[\int_{r=\epsilon} n_{c} C_{a b}^{c} S^{a b}-\int_{r>\epsilon} S^{a b} \nabla_{c} C_{a b}^{c}\right]
\end{array}
$$

However, for $r \neq 0$ it follows from equations (15) and (13) that $\nabla_{c} C_{a b}^{c}=0$. Furthermore, we have $n_{c}=-\nabla_{c} r$. Then using equation (15) we find

$$
\begin{array}{r}
\tilde{R}_{a b}\left[S^{a b}\right]=\lim _{\epsilon \rightarrow 0} \int_{r=\epsilon} \sin ^{2} \gamma r^{-3} \psi_{a} \psi_{b} S^{a b} \\
=\sin ^{2} \gamma \lim _{r \rightarrow 0} \int_{-\infty}^{\infty} d t \int_{-\infty}^{\infty} d z \int_{0}^{2 \pi} d \phi\left(\sin ^{2} \phi S^{x x}+\cos ^{2} \phi S^{y y}-\sin \phi \cos \phi\left(S^{x y}+S^{y x}\right)\right) \\
=\pi \sin ^{2} \gamma \int_{-\infty}^{\infty} d t \int_{-\infty}^{\infty} d z\left(S^{x x}+S^{y y}\right)
\end{array}
$$


where the last line is evaluated at $r=0$. That is, we have

$$
\tilde{R}_{a b}=\pi \sin ^{2} \gamma \delta(x) \delta(y)\left(\partial_{a} x \partial_{b} x+\partial_{a} y \partial_{b} y\right)
$$

We now calculate the Einstein tensor of $\tilde{g}_{a b}$. Specializing equation (5) to the metric $\tilde{g}_{a b}$ and using equations (13), (14) and (15) we find

$$
\tilde{G}_{a b}=\tilde{R}_{a b}-\frac{1}{2} \nabla_{c}\left(\tan ^{2} \gamma r^{-1} \nabla^{c} r \tilde{g}_{a b}\right)
$$

Note that the quantity in parentheses is locally integrable and thus defines a distribution. Equation (22) therefore makes sense as a distributional equation whose meaning is that for any test tensor field $S^{a b}$ we have

$$
\tilde{G}_{a b}\left[S^{a b}\right]=\tilde{R}_{a b}\left[S^{a b}\right]+\frac{1}{2} \int_{M} \tan ^{2} \gamma r^{-1} \nabla^{c} r \tilde{g}_{a b} \nabla_{c} S^{a b} .
$$

A calculation analogous to equation (20) then yields

$$
\tilde{G}_{a b}=\pi \tan ^{2} \gamma \delta(x) \delta(y)\left[\partial_{a} t \partial_{b} t-\partial_{a} z \partial_{b} z-\frac{1}{2} \sin ^{2} \gamma\left(\partial_{a} x \partial_{b} x+\partial_{a} y \partial_{b} y\right)\right] .
$$

Thus the Einstein tensor of Minkowski spacetime minus a wedge makes sense as a distribution.

However, in reference [13] Geroch and Traschen consider one parameter families of smooth metrics that tend to Minkowski spacetime minus a wedge. They show that the limitting value of the mass per unit length depends not only on the deficit angle, but also on which one parameter family is used. Futamase and Garfinkle [35] consider one parameter families of self-gravitating Abelian-Higgs strings. Here too, the spacetime tends to Minkowski spacetime minus a wedge, and the relation between mass per unit length and deficit angle depends on which one parameter family is used. Thus there is a physically relevant sense in which Minkowski spacetime minus a wedge does not have a distributional stress energy.

How are we to reconcile the results of this section with those of references [13] and [35] on the behavior of one parameter families of spacetimes tending to Minkowski spacetime minus a wedge? The answer has to do with the notion of convergence of a one parameter family of metrics. Reference 13] introduces a convergence condition on a sequence of regular metrics and shows that if a sequence of metrics satisfies this convergence condition, then a limit curvature tensor exists and is the curvature tensor of the limit metric. If there is an analogous convergence condition for semi-regular metrics, then the one parameter families of cosmic strings considered in references [13] and [35] do not satisfy it. Thus, though we have found a distributional stress energy tensor for Minkowski spacetime minus a wedge, this distribution may not be an appropriate physical description of the energy content of the string.

In this section, we have considered only the metric for a long straight string. However, the metric of a thin circular loop of cosmic string, momentarily at rest, has been found by Frolov, Israel and Unruh [36]. Also, a general description of the behavior of curved thin strings has been given by Unruh, Hayward, Israel and McManus [37]. It would be interesting to see whether these metrics are semi-regular, and if so to calculate their distributional curvature.

\section{TRAVELING WAVE}

We now consider traveling waves with distributional curvature. Let $g_{a b}$ be a smooth metric with null, hypersurface orthogonal Killing vector $k^{a}$. Then there is a scalar $A$ such that $\nabla_{a} k_{b}=k_{[b} \nabla_{a]} A$. Consider the metric $\tilde{g}_{a b}=$ $g_{a b}+F k_{a} k_{b}$ where $F$ is a scalar satisfying $k^{a} \nabla_{a} F=0$. The physical interpretation of the metric $\tilde{g}_{a b}$ is a wave traveling on the background metric $g_{a b}$ without changing its amplitude or shape.

As shown by Garfinkle and Vachaspati [14], the Ricci tensor of the traveling wave satisfies

$$
\left(\tilde{g}^{-1}\right)^{a c} \tilde{R}_{c b}=R_{b}^{a}-\frac{1}{2} k^{a} k_{b} e^{-A} \nabla_{c} \nabla^{c}\left(e^{A} F\right) .
$$

Here indicies are lowered and raised with $g_{a b}$ and its inverse. Since this expression is linear in $F$, one might hope that the Ricci tensor makes sense as a distribution in some cases where $F$ is not smooth.

We choose as the background metric $g_{a b}$ for the traveling waves, Minkowski spacetime expressed as

$$
d s^{2}=2 d u d v+d r^{2}+r^{2} d \phi^{2} .
$$


Traveling wave metrics with Minkowski spacetime as a background are called plane fronted waves. [38] In what follows, $g_{a b}$ and its inverse are used to lower and raise indicies, the volume form of $g_{a b}$ is used to convert tensor fields into tensor densities and the derivative operator $\nabla_{a}$ compatible with $g_{a b}$ is used to define the Christoffel tensor and the curvature of equations (1) and (2). The coordinates $(u, v, r, \phi)$ are related to the usual Cartesian coordinates $(t, x, y, z)$ by $u=t-z, v=-(t+z) / 2, x=r \cos \phi$ and $y=r \sin \phi$. Our null Killing field is $k_{a}=\nabla_{a} u$. This $k^{a}$ is covariantly constant, so $A=0$. We consider the traveling wave metric given by

$$
\tilde{g}_{a b}=g_{a b}+f(u) \ln r k_{a} k_{b}
$$

where $f(u)$ is any smooth function.

For any test field $S^{a b}$ we have

$$
\tilde{g}_{a b} S^{a b}=S+\ln r f S^{u u} .
$$

It then follows that $\tilde{g}_{a b}$ is locally integrable and defines a distribution. Paralleling the treatment of equation (12) we find that the weak derivative of $\tilde{g}_{a b}$ is given for $r \neq 0$ by

$$
\nabla_{c} \tilde{g}_{a b}=\left(\ln r f^{\prime} k_{c}+\frac{f}{r} \nabla_{c} r\right) k_{a} k_{b}
$$

Note that this weak derivative is integrable, but not square integrable. It then follows that $\tilde{g}_{a b}$ is not regular. (The lack of regularity of $\tilde{g}_{a b}$ also follows from the fact that $\tilde{g}_{a b}$ is not locally bounded).

From equations (11) and (29) it follows that the Christoffel tensor is

$$
C_{a b}^{c}=\frac{f^{\prime} \ln r}{2} k_{a} k_{b} k^{c}+\frac{f}{2 r}\left(\left[k_{a} \nabla_{b} r+k_{b} \nabla_{a} r\right] k^{c}-k_{a} k_{b} \nabla^{c} r\right) .
$$

This tensor is locally integrable and defines a distribution. In addition, $C_{c b}^{c}=0$ and $C_{m[b}^{d} C_{a] c}^{m}=0$. It then follows that $\tilde{g}_{a b}$ is semi-regular.

From equation (16) and the properties of $C_{a b}^{c}$ we have

$$
\tilde{R}_{a b}\left[S^{a b}\right]=-C_{a b}^{c}\left[\nabla_{c} S^{a b}\right]
$$

for any test field $S^{a b}$. Then a calculation analogous to that of equations (19) and (20) leads to

$$
\tilde{R}_{a b}=\pi f \delta(x) \delta(y) \partial_{a} u \partial_{b} u
$$

Thus the Ricci tensor is a distribution concentrated at $r=0$. Using equation (30) in equation(5) one can show that the Einstein tensor is also defined as a distribution, and, in fact, that $\tilde{G}_{a b}=\tilde{R}_{a b}$. Therefore, the stress-energy of this traveling wave is a distribution.

Though we have found a distributional Einstein tensor, and thus, through Einstein's equation, a distributional stress-energy tensor for a semi-regular traveling wave metric, it is not clear what the physical meaning of this stressenergy tensor is. To clarify this issue, it would be helpful to find a one parameter family of metrics approaching the metric of equation (27) and see whether their Einstein tensors approach the distribution of equation (32).

In this section, we have treated only one traveling wave metric. However, traveling waves have been found on anti de Sitter space, [39] Melvin's Magnetic Universe [40] and certain solutions of low energy string theory. [41] Some of these metrics are semi-regular, but not regular. Study of these metrics could shed further light on the nature of distributional curvature in general relativity.

\section{ACKNOWLEDGEMENTS}

I would like to thank Jennie Traschen for helpful discussions. I would also like to thank the Institute for Theoretical Physics at Santa Barbara (partially supported by NSF grant PHY94-07194) for hospitality. This work was partially supported by NSF grant PHY-9722039 to Oakland University. 
[1] J. Columbeau, Journal of Mathematical Analysis and Applications, 94, 96 (1983)

[2] J. Columbeau, Bulletin of the American Mathematical Society, 23, 251 (1990)

[3] C. Clarke, J. Vickers and J. Wilson, Class. Quantum Grav. 13, 2485 (1996)

[4] J. Vickers and J. Wilson, Class. Quantum Grav. 16, 579 (1999)

[5] J. Vickers and J. Wilson, gr-qc/9807068

[6] W. Israel, Nuovo Cimento B 44, 1 (1966)

[7] W. Israel, Phys. Rev. D15, 935 (1977)

[8] A. Taub, J. Math. Phys. 21, 1423 (1980)

[9] C. Clarke and T. Dray, Class. Quantum Grav. 4, 265 (1987)

[10] J. Griffiths, Colliding Plane Waves in General Relativity (Oxford University Press, Oxford 1991)

[11] C. Barrabes and W. Israel, Phys. Rev. D43, 1129 (1991)

[12] C. Barrabes and P. Hogan, Phys. Rev. D58, 044013 (1998)

[13] R. Geroch and J. Traschen, Phys. Rev. D36, 1017 (1987)

[14] D. Garfinkle and T. Vachaspati, Phys. Rev. D42, 1960 (1990)

[15] A. Taub, Ann. Phys. (N.Y.) 134, 326 (1981)

[16] T. Dray, Int. J. Mod. Phys. D6, 717 (1997)

[17] G. Ellis, A. Sumeruk, D. Coule and C. Hellaby, Class. Quantum Grav. 9, 1535 (1992)

[18] G. Ellis, Gen. Rel. Grav. 24, 1047 (1992)

[19] T. Dray, J. Math. Phys. 37, 5627 (1996)

[20] S. Hayward, Class. Quantum Grav. 9, 1851 (1992). Erratum: Class. Quantum Grav. 9, 2453 (1992)

[21] F. Embacher, Phys. Rev. D51, 6764 (1995)

[22] T. Dray, C. Manogue, and R. Tucker, Phys. Rev. D48, 2587 (1993)

[23] C. Hellaby and T. Dray, Phys. Rev. D49, 5096 (1995)

[24] T. Dray and C. Hellaby, Phys. Rev. D52, 7333 (1995)

[25] T. Dray and C. Hellaby, J. Math. Phys. 35, 5922 (1994)

[26] T. Dray, C. Manogue and R. Tucker, Class. Quantum Grav. 12, 2767 (1995)

[27] M. Carfora and G. Ellis, Intl. J. Mod. Phys. D4, 175 (1995)

[28] T. Dray, G. Ellis, C. Hellaby and C. Manogue, Gen. Rel. Grav. 29, 591 (1997)

[29] S. Hayward, Phys. Rev. D52, 7311 (1995)

[30] J. Martin, Phys. Rev. D52, 6708 (1995)

[31] F. Embacher, Class. Quantum. Grav. 13, 921 (1996)

[32] M. Kriele and J. Martin, Class. Quantum Grav. 12, 503 (1995)

[33] S. Deser, R. Jackiw and G. 'tHooft, Ann. Phys. 152, 220 (1984)

[34] S. Deser and R. Jackiw, Ann. Phys. 153, 405 (1984)

[35] T. Futamase and D. Garfinkle, Phys. Rev. D37, 2086 (1988)

[36] V. Frolov, W. Israel and W. Unruh, Phys. Rev. D39, 1084 (1989)

[37] W. Unruh, G. Hayward, W. Israel and D. McManus, Phys. Rev. Lett. 62, 2897 (1989)

[38] D. Kramer, H. Stephani, M. MacCallum and E. Herlt, (1980) Exact Solutions of Einstein's Field Equations (Cambridge University Press, Cambridge).

[39] S. Siklos, (1985) in Galaxies, Axisymmetric Systems and Relativity, M. MacCallum, Ed. (Cambridge University Press, Cambridge)

[40] D. Garfinkle and M. Melvin, Phys. Rev. D45, 1188 (1992)

[41] D. Garfinkle, Phys. Rev. 46, 4286 (1992) 